\title{
Multimodal texture analysis of OCT images as a diagnostic application for skin tumors
}

\author{
Dmitry S. Raupov ${ }^{1 *}$, Oleg O. Myakinin ${ }^{1}$, Ivan A. Bratchenkoํ, Valery P. Zakharov ${ }^{1}$, \\ and Alexander G. Khramov ${ }^{2}$ \\ ${ }^{1}$ Dept. of Laser and Biotechnical Systems, Samara National Research University, Russian Federation \\ ${ }^{2}$ Technical Cybernetics Dept., Samara National Research University, Russian Federation \\ * e-mail: radim94@mail.ru
}

\begin{abstract}
Optical coherence tomography (OCT) is an effective tool for determination of pathological topology that reflects structural and textural metamorphoses of tissue. In this paper, we propose a report about our examining of the validity of OCT in identifying changes using a skin cancer texture analysis compiled from Haralick texture features, fractal dimension, complex directional field features and Markov random field method from different tissues. The experimental data set contains 530 OCT images with normal skin and tumors as Basal Cell Carcinoma (BCC), Malignant Melanoma (MM) and Nevus. Speckle reduction is an essential pre-processing part for OCT image analyze. In this work, we used an interval type-II fuzzy anisotropic diffusion algorithm for speckle noise reduction in OCT images (B- and/or C-scans). The Haralick texture features as contrast, correlation, energy, and homogeneity have been calculated in various directions. A boxcounting method and other methodologies have been performed to evaluate fractal dimension of skin probes. The complex directional field calculated by the local gradient methodology provides important data for linear dividing of species. We also estimated autocorrelation function using Markov random fields. Additionally, the boosting has been used for the quality enhancing of the diagnosis method. And, finally, artificial neural network (ANN) has been utilized for comparing received rates. Our results demonstrate that these texture features may present helpful information to discriminate a tumor from healthy tissue. We obtained sensitivity about $92 \%$ and specificity about $95 \%$ for a task of discrimination between MM and healthy skin. Finally, a universal four classes classificatory has been built with average accuracy 75\%. (C) 2017 Journal of Biomedical Photonics \& Engineering.
\end{abstract}

Keywords: optical coherence tomography; texture analysis; Haralick features; fractal dimension; complex directional field; diffuse filter; Markov random field.

Paper \#3175 received 23 Mar 2017; revised manuscript received 25 Apr 2017; accepted for publication 25 Apr 2017; published online 29 Apr 2017. doi: 10.18287/JBPE17.03.010307. [Special Issue. Years in Biophotonics: 70th Anniversary of Prof. A.V. Priezzhev].

\section{References}

1. D. Huang, E. A. Swanson, C. P. Lin, J. S. Schuman, W. G. Stinson, W. Chang, M. R. Hee, T. Flotte, K. Gregory, C. A. Puliafito, and J. G. Fujimoto, "Optical coherence tomography," Science 254(5035), 1178-1181 (1991).

2. A. F. Fercher, W. Drexler, C. K. Hitzenberger, and T. Lasser, "Optical coherence tomography-principles and applications," Rep. Prog. Phys. 66(2), 239-303 (2003).

3. A. M. Sergeev, L. S. Dolin, and D. N. Reitze, "Optical tomography of biotissues: past, present and future," Optics\&Photonics News 12(7), 28-35 (2001). 
4. E. A. Genina, S. A. Kinder, A. N. Bashkatov, and V. V. Tuchin, "Liver images contrasting in optical coherence tomography with help of nanoparticles," Proceedings of Saratov University 11(2), 10-13 (2011).

5. Y. Freund, and R. E. Schapire, "A Decision-Theoretic Generalization of On-Line Learning and an Application to Boosting," J. of Computer and System Sciences 55(1), 119-139 (1997).

6. S. Wang, M. Singh, A. L. Lopez, C. Wu, R. Raghunathan, A. Schill, J. Li, K. V. Larin, and I. V. Larina, "Direct four-dimensional structural and functional imaging of cardiovascular dynamics in mouse embryos with 1.5 MHz optical coherence tomography," Opt. Lett. 40(20), 4791-4794 (2015).

7. V. P. Zakharov, K. Larin, and I. A. Bratchenko, "Increasing the information content of optical coherence tomography skin pathology detection," Proceedings of SSAU 26, 232-239 (2011).

8. V. P. Zakharov, I. A. Bratchenko, V. I. Belokonev, D. V. Kornilin, and O. O. Myakinin, "Complex optical characterization of mesh implants and encapsulation area,” J. Innov. Opt. Health Sci. 06, 1350007 (2013).

9. P. Puvanathasan, and K. Bizheva, "Interval type-II fuzzy anisotropic diffusion algorithm for speckle noise reduction in optical coherence tomography images," Optics Express 17(2), 733-746 (2009).

10. H. Tizhoosh, "Image thresholding using type II fuzzy sets," Pattern Recognition 38(12), 2363-2372 (2005).

11. M. Wojtkowski, "High-speed optical coherence tomography: basics and applications," Applied Optics 49(16), 30-61 (2010).

12. A. M. Forsea, E. M. Carstea, L. Ghervase, C. Giurcaneanu, and G. Pavelescu, "Clinical application of optical coherence tomography for the imaging of non-melanocytic cutaneous tumors: a pilot multi-modal study," Journal of medicine and Life 3(4), 381-389 (2010).

13. N. Sarkar, and B. B. Chaudhuri, "An efficient approach to estimate fractal dimension of textural images," Pattern Recognition 25(9), 1035-1041 (1992).

14. J. Huang, and D. Turcotte, "Fractal image analysis: application to the topography of Oregon and synthetic images," J. Opt. Soc. Am. 7(6), 1124-1130 (1990).

15. Y.-Z. Liu, F. A. South, Y. Xu, P. S. Carney, and S. A. Boppart, "Computational optical coherence tomography [Invited]," Biomed. Opt. Express 8, 1549-1574 (2017)

16. A. I. Plastinin, and A. V. Kupriyanov, "A model of Markov random field in texture image synthesis and analysis," Proceedings of Samara State Aerospace University 2, 252-257 (2008).

17. N. U. Ilyasova, A. V. Ustinov, and A. G. Khramov, "Numerical methods and algorithms of building of directional fields in quasiperiodic structures," Computer Optics 18, 150-164 (1998).

18. R. E. Schapire, Y. Freund, P. Bartlett, and W. S. Lee, "Boosting the margin: A new explanation for the effectiveness of voting methods," Annals of Statistics 26(5), 1651-1686 (1991).

19. O. O. Myakinin, V. P. Zakharov, I. A. Bratchenko, D. V. Kornilin, and A. G. Khramov, “A complex noise reduction method for improving visualization of SD-OCT skin biomedical images," Proc. SPIE 9129, 91292Y, (2014).

20. R. M. Haralick, K. Shanmugam, and I. Dinshtein, "Textural features for image classification," IEEE Trans. Syst. Man Cybern. 3(6), 610-621 (1973).

21. R. M. Haralick, and L. G. Shapiro, Computer and Robot Vision: Vol. 1, Addison-Wesley (1992). ISBN: 9780201108774.

22. C. Flueraru, D. P. Popescu, Y. Mao, S. Chang, and M. G. Sowa, "Added soft tissue contrast using signal attenuation and the fractal dimension for optical coherence tomography images of porcine arterial tissue," Physics in Medicine and Biology 55(8), 2317-2331 (2010).

23. A. C. Sullivan, J. P. Hunt, and A. L. Oldenburg, "Fractal analysis for classification of breast carcinoma in optical coherence tomography," Journal of Biomedical Optics 16(6), 066010 (2013).

24. W. Gao, Improving the quantitative assessment of intraretinal features by determining both structural and optical properties of the retinal tissue with optical coherence tomography, Ph.D. thesis (2012).

25. R. F. Voss, "Random fractal forgeries" in: Fundamental Algorithms for Computer Graphics, R. A. Earnshaw (ed.), Springer-Verlag, Berlin, 805-835 (1985).

26. A. Annadhason, "Methods of Fractal Dimension Computation," IRACST 2(1), 166-169 (2012).

27. N. Sarkar, and B. B. Chaudhuri, "An Efficient Differential Box-Counting Approach to Compute Fractal Dimension of Image,” IEEE Trans. Syst. Man Cybern. 24(1), 115-120 (1994).

28. J. B. Florindo, and O. M. Bruno, "Fractal Descriptors in the Fourier Domain Applied to Color Texture Analysis," Chaos 21(4), 043112 (2011).

29. H. Ahammer, "Higuchi Dimension of Digital Images," PLoS ONE 6(9), e24796 (2011).

30. G. Winkler, Image Analysis, Random Fields and Dynamic Monte Carlo Methods, Springer, Verlag (1995). ISBN: 978-3-642-97522-6.

31. A. I. Plastinin, The method of formation of textural features based on Markov models, Ph.D. thesis, 04200201565, 30-45 (2012).

32. J. Schmidhuber, "Deep learning in neural networks: An overview," Neural Networks 61, 85-117 (2015).

33. D. Rutkovskaya, M. Pilinsky, and L. Rutkowski, Neural networks, genetic algorithms and fuzzy systems, Telecom, 5-20 (2006). 
34. S. Haykin, Neural Networks: A Comprehensive Foundation Paperback, MacMillan Publishing Company, 30-40 (1994).

35. W. McCulloch, and W. Pitts, "A Logical Calculus of Ideas Immanent in Nervous Activity," Bulletin of Mathematical Biophysics 5(4), 115-133 (1943).

36. M. F. Moller, “A Scaled Conjugate Gradient Algorithm for Fast Supervised Learning,” Neural Networks 6(4), 525-533 (1993).

37. K. V. Vorontsov, "About the problem-oriented bases optimization of recognition problem," Computational Mathematics and Mathematical Physics 38(5), 870-880 (1998).

38. K. V. Vorontsov, "Optimization methods for linear and monotone correction in the algebraic approach to the recognition problem," Computational Mathematics and Mathematical Physics 40(1), 166-176 (2000).

39. L. Breinman, Classification and Regression Trees, Chapman\&Hall, Boca Raton (1993).

40. R. O. Duda, P. E. Hart, and D. G. Stork, Pattern Classification, Wiley (2001).

41. D. S. Raupov, O. O. Myakinin, I. A. Bratchenko, D. V. Kornilin, V. P. Zakharov, and A. G. Khramov, "Skin cancer texture analysis of OCT images based on Haralick, fractal dimension and the complex directional field features," Proc. SPIE 9887, 98873F (2016).

42. D. S. Raupov, O. O. Myakinin, I. A. Bratchenko, D. V. Kornilin, V. P. Zakharov, and A. G. Khramov, "Skin cancer texture analysis of OCT images based on Haralick, fractal dimension, Markov random field features, and the complex directional field features," Proc. SPIE 10024, 100244I (2016).

43. T. Gambichler, M. H. Schmid-Wendtner, I. Plura, P. Kampilafkos, M. Stücker, C. Berking, and T. Maier, "A multicentre pilot study investigating high-definition optical coherence tomography in the differentiation of cutaneous melanoma and melanocytic naevi," Journal of the European Academy of Dermatology and Venereology 29(3), 537-541 (2015).

44. C. Wahrlich, S. A. Alawi, S. Batz, J. W. Fluhr, J. Lademann, and M. Ulrich, "Assessment of a scoring system for Basal Cell Carcinoma with multi-beam optical coherence tomography," Journal of the European Academy of Dermatology and Venereology 29(8), 1562-1569 (2015).

45. V. P. Zakharov, I. A. Bratchenko, D. N. Artemyev, O. O. Myakinin, D. V. Kornilin, S. V. Kozlov, and A. A. Moryatov, "Comparative analysis of combined spectral and optical tomography methods for detection of skin and lung cancers," Journal of Biomedical Optics 20(2), 025003 (2015).

46. Q. Abbas, M. E. Celebi, C. Serrano, I. Garci'a, G. Ma, "Pattern classification of dermoscopy images: A perceptually uniform model," Pattern Recognition 46(1), 86-97 (2013).

\section{Introduction}

Optical Coherence Tomography (OCT) is a great tool for contactless nondestructive study of optically inhomogeneous mediums [1]. Recently, effective application of OCT has been confirmed in various directions of clinical practice such as gastroenterology, urology, dermatology, gynecology, ophthalmology, otolaryngology, stomatology and others [2, 3]. OCT has a position as a non-invasive method of visualization of inner structure of optically heterogeneous objects based on a principle of low-coherence interferometry using near infrared range $(0.75-1.3 \mu \mathrm{m})$ as light source. OCT visualizes an inner microstructure of skin down to $2 \mathrm{~mm}$ with space resolution 10-15 $\mu \mathrm{m}$ without human tissue invasion [4]. Optical coherence tomography is usually employed for a measurement of structural changes of tissue. The possibility of OCT in detecting changes using Haralick's texture features, fractal dimension and complex directional field extracted from different tissues has been investigated in this paper.

The main goal of this work is the development of a multimodal method for the texture analysis of OCT images. As is well known, one of the most dangerous neoplasms, the malignant melanoma, has a large absorption coefficient, which means the OCT image has a very low level of signal-to-noise ratio (SNR) in the region of the neoplasm. Thus, using only one textural technique is a priory not enough. However, combining texture modalities can guarantee accuracy improvement $[5]$.

Any coherent method implies speckle noise impact for the imaging. In general case, the noise can be removed by digital filter from final OCT volume after a mandatory PC processing [6]. On a 2D slice of tissue volume in vertical (B-scan) or horizontal (C-scan) planes, it is available to see dynamics of anisotropic growth for malicious area and following deformation for encircling healthy one. It is just an image in terms of Digital Image Processing and the denoising by way of a general image processing filter [7, 8] must be applied. We used an interval type-II fuzzy anisotropic diffusion algorithm $[9,10]$ for speckle noise reduction in OCT images for this research as more reliable for processing on OCT images.

Haralick texture features have been evaluated in different directions as an initial and basic solution for the multimodal approach. These quickly computable features contain useful information for categorizing, classifying diagnostic images as B-scans. However, in biology and medicine, the shapes of structures such as molecules, cells, tissues and organs also play an important role in the diagnosis of diseased tissue [11, 12]. Fractal dimension could also distinguish the structural changes of tissue. Quantitative evaluation of 
the fractal dimension could be an efficient concept to differ tumor tissue from normal healthy tissue [13, 14]. So the structural changes in fractal dimension may give further information observing cellular layers and injury in skin pathology. Besides, the using of the OCT techniques contributes to the application of fractal analysis in the diagnostics of skin cancer. The suspicious skin tissue could be revealed by accomplishment of fractal analysis for certain biological structures in OCT images. Thus, fractal analysis with the OCT imaging techniques could give an efficient diagnostic methodology to classify tumors as BCC and MM [15]. Also, it can be used for differentiation between nevus and healthy skin probes. Usually, we can simplify numerical dependencies in tissue topology elementary volumes (planes, specifically, if we are talking about images) by suggestion, that volume depends from only her neighbor ones. It is means, that a computing, for instance, Markov random fields' [16] features could be helpful to provide us useful information about nature of tumor.

We included a method of the complex directional field [17] to this features' list to improve the characteristics' quality of tumor recognition. Malignant tissue growth anisotropically, which means it may be interpreted by the complex directional field calculated from a C-scan. General features like variance and correlation could be used for dividing scans with tumor and healthy probes. A basic idea of a directional field application is an attempt to analyze the directions of anisotropic growth of malicious skin and possibility of tissue further development. High relevant results on quasiregular structures such as interferograms, fingerprints, crystallograms and many others show us its universality [17]. The complex form provides more evaluation precision and a relationship between a directional field and a weight function. The C-scan analyzing gives us an ability to appreciate other planes of spreading pathology on the cellular layers.

Unfortunately, we also should take into account additional a priory information about preliminary diagnosis when we use textural features for common dividing between two classes (MM or BCC, MM or Nevus etc.). Thus, it could be a big challenge for general practitioner. So, it is very important to have a universal classifier for accurate definition between many tumor classes. A priory information is also required for this case, but preliminary diagnosis could be much less accurate. The boosting [18] is used in this work with aim to generalize all textural features, enhance diagnosis quality and receive classifier for discriminating between 4 classes (MM, BCC, Nevus or healthy skin). Additionally, we tested ANN as an alternative method of machine learning to create a linear combination of described textural features for increasing sensitivity and specificity.

\section{Materials and Methods}

\subsection{OCT-setup scheme and tissue samples}

The OCT system (Fig. 1) comprises of a broadband superluminescent laser diode $(840 \pm 45 \mathrm{~nm}$ wavelength range, $14 \mathrm{~mW}$ output power) at the source end, Michelson interferometer with 50/50 split ratio to the sample and reference arms and a spectrometer at the detector end. The spectrometer comprises of a diffraction grating (1200 grooves $/ \mathrm{mm}$ ) and a CCD line scan camera (4096 pixel resolution, $29.3 \mathrm{kHz}$ line rate). The interference signal from the sample and the reference arms of the Michelson interferometer is detected by the spectrometer and digitized by an image acquisition card (NI-IMAQ PCI-1428). Depth profile (A-line) is obtained by converting the interference signal detected by the IMAQ into linear k-space [19]. The imaging axial and lateral resolution of the OCT system is about $6 \mu \mathrm{m}$ in biological tissue.

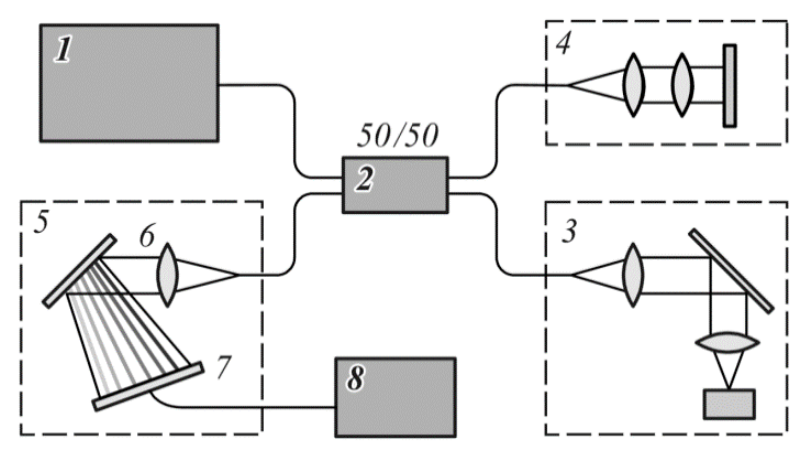

Fig. 1 Spectral domain OCT scheme: 1 - broadband source, 2 - 50/50 beam splitter, 3 - sample arm, 4 - reference arm, 5 spectrometer with grating 6 and CCD camera 7, 8 - computer with IMAQ.

The experimental data set contains 530 OCT images with normal skin and tumors as Basal Cell Carcinoma (BCC), Malignant Melanoma (MM) and Nevus. The institutional Review Board of Samara National Research University approved the study protocol. This research adhered to the tenets set forth in the Declaration of Helsinki. Informed consent of each subject was obtained.

\subsection{Haralick features}

For evaluating Haralick features, a gray-level cooccurrence matrix (GLCM) from image $I$ should be calculated. GLCM is based on frequency evaluating of a pixel with gray-level value $i$ horizontally (vertically, diagonally) connected to a pixel with the value $j$. Each element $(i, j)$ in GLCM specifies the number of times that the pixel with value $i$ occurred horizontally adjacent to a pixel with value $j[20]$. 


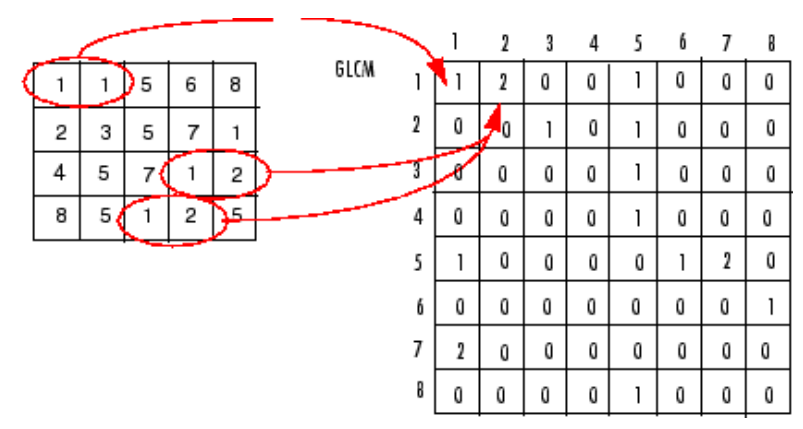

Fig. 1 A gray-level co-occurrence matrix sketch [21].

For calculating Haralick's features, image is represented as a grate of pixels of mandatory intensity, which is used for calculating four GLCMs of relative frequencies of pixels' order in directions $0^{\circ}, 45^{\circ}, 90^{\circ}$, $135^{\circ}$. Textural features based on these matrixes are used for images classification as will be described later.

Homogeneity returns a value that measures the closeness of the distribution of elements in the GLCM to the diagonal GLCM.

$$
f_{1}=\sum_{i, j} p(i, j) .
$$

Correlation serves as a measure of dependency of neighboring pixels over the whole image.

$$
f_{3}=\sum_{i, j} \frac{(i-\mu i)(j-\mu j) p(i, j)}{\sigma_{i} \sigma_{j}}
$$

Variance defines a measure of the intensity contrast between a pixel and its neighbor over the whole image.

$$
f_{4}=\sum_{i, j}|i-j|^{2} p(i, j) .
$$

Energy is calculated as the sum of squared elements in the GLCM.

$$
f_{5}=\sum_{i, j} \frac{p(i, j)}{1+|i-j|}
$$

\subsection{Fractal analysis}

In the analysis of OCT images, fractal analysis has been used to examine the structural change of biological tissue. For example, Fluearu [22] utilized the box counting method to compute the fractal dimension to characterize porcine arterial tissue. Sullivan [23] used the box counting method to evaluate the fractal dimension to detect the breast carcinoma. Gao [24] applied the power spectrum method to carry out the fractal analysis on the layered retinal tissue for diagnosing the diabetic retinopathy.

The most popular algorithm for computing the fractal dimension of one dimensional and two dimensional data is the box counting, method originally developed by Voss [25]. In this method, the fractal surface is covered with a grid of $n$-dimensional boxes or hyper-cubes with side length, $\varepsilon$ and counting the number of boxes that contains a part of the fractal $N(\varepsilon)$. As for signals, the grid consists of squares and for images, the grid consists of cubes. The fractal surface is covered with boxes of recursively different sizes. An input signal with $N$ elements or an image of size $N * N$ is used as input where $N$ is a power of 2 [26].

$$
D_{b c}=\frac{\log N(\varepsilon)}{\log \frac{1}{\varepsilon}}
$$

Other method for counting fractal dimension is differential box-counting method [27]. In this method, $N$ is counted in other way. Consider that the image of size $M \times M$ pixels has been scaled down to a size $s \times s$, where $M / 2 \geq s \geq 1$ and $s$ is an integer. Then we have an estimate of $\varepsilon=s / M$. Now, as in previous techniques, consider the image as a 3-D space with $(x, y)$ denoting 2-D position and the third coordinate (z) denoting gray level. The $(x, y)$ space is partitioned into grids of size $s \times s$. On each grid there is a column of boxes of size $\times s \times s^{\prime}$. If the total number of gray levels is $G$ then $G / s^{\prime}=M / s$. For example, see Fig. 3, where $s=s^{\prime}=3$. Assign numbers 1,2 ,..to the boxes as shown. Let the minimum and maximum gray level of the image in the $(i, j)$ th grid fall in box number $k$ and $l$, respectively. In this approach

$$
n_{r}(i, j)=l-k+1
$$

is the contribution of $N_{r}$, in $(\mathrm{i}, \mathrm{j})$ th grid. For example, in Fig. $3, n_{r}(i, j)=3-1+1$. Taking contributions from all grids, we have

$$
N_{r}=\sum_{i, j} n_{r}(i, j) .
$$

$N_{r}$ is counted for different values of $r$, i.e., different values of $s$. Then using (7), it is possible to estimate $D$, the fractal dimension, from the least square linear fit of $\log N_{r}$ against $\log (1 / r)$.

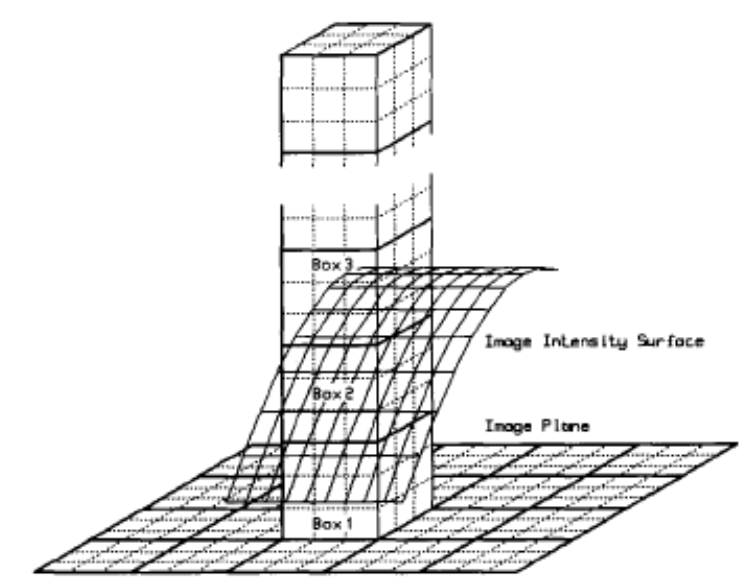

Fig. 3 Determination of $n_{r}$ by differential box-counting method [28]. 
Also we used power-spectrum method for counting fractal dimension [29]. This method is an application of the Fourier power spectrum method. The real-space image is Fourier transformed by means of the fast Fourier transform and the power spectrum, $P_{i}$ is computed as:

$$
P_{i}=\operatorname{Re}\left(k_{i}\right)^{2}+\operatorname{Im}\left(k_{i}\right)^{2}
$$

Then the power spectrum of an ideal one dimensional fractal signal with dimension $D$ is considered. This has the formula

$$
P_{i}=c k_{i}^{-\beta}
$$

where $c$ is a constant and $\beta$ is the spectral exponent. The index $\beta$ is related to the Fourier transform dimension $D_{F}$. The value of the spectral $\beta$ and $D_{F}$, can be found out for the input signal by fitting a least squares error line to the data. The merits of this approach are it is generalizable, potentially more accurate and the computation of $D_{F}$ is based on an explicit formula [23].

\subsection{The complex directional field}

Complex directional field defines as:

$$
\dot{\varphi}(x, y)=w(x, y) \exp (i 2 \varphi(x, y))
$$

where $w(x, y)$ has a physical meaning as reliability of directional field in this point [17].

Methods of local gradients are based on the fact, that function's gradient in each point is perpendicular to tangency of contour line in this point. These methods are based on evaluation of gradient intensity function for different positions of local mask inside scanned on image outer window $W$ with size $M * N$ (local gradient) $\left(f_{x}^{k, l}, f_{y}^{k, l}\right)$, where $1 \leq k \leq N, 1 \leq l \leq M$.

$$
\begin{gathered}
\tan \hat{\varphi}(x, y)=-\frac{f_{x}}{f_{y}}, 0 \leq \hat{\varphi}(x, y) \leq \pi \\
\left(f_{x}, f_{y}\right)=\left(\frac{\partial I(x, y)}{\partial x}, \frac{\partial I(x, y)}{\partial y}\right) .
\end{gathered}
$$

The local gradient methodology is divided into two classes. First one is methods of gradient projections averaging and second one is methods of local direction angles averaging. Method of gradient projections averaging is based on using of local gradients, mandatory to position $(k, l)$ of local mask in calculating of intensity function gradient in the center of outer window $W$ :

$$
\left(f_{x}, f_{y}\right)=\frac{1}{N M} \sum_{k=1}^{N} \sum_{l=1}^{M}\left(f_{x}^{k, l}, f_{y}^{k, l}\right) .
$$

Method of local direction angles averaging uses local gradients $\left(f_{x}^{k, l}, f_{y}^{k, l}\right)$ for calculation of local angles:

$$
\varphi_{k, l}=-\tan ^{-1}\left(\frac{f_{x}^{k, l}}{f_{y}^{k, l}}\right) .
$$

Then direction of trace in the center of outer window $W$ can be evaluated by averaging of local angles field:

$$
\hat{\varphi}=\frac{1}{2} \arg \sum_{k=1}^{N} \sum_{l=1}^{M} \exp \left(i 2 \varphi_{k, l}\right) .
$$

The value of a weight function of a direction field will be next:

$$
\widehat{w}=\left|\frac{1}{M N} \sum_{k=1}^{N} \sum_{l=1}^{M} \exp \left(i 2 \varphi_{k, l}\right)\right| .
$$

\subsection{Markov random fields}

A Markov random field, Markov network or undirected graphical model is a graphical model in which a set of random variables have a Markov property described by an undirected graph [30]. Connection of nodes with each other is defined by localities system as $N=$ $\left\{N_{S} \mid s \in S\right\}$, where $N_{S}$ is a neighbors set of node $s, S$ is finite set of nodes. Localities system has property: $s \notin N_{s}$. Random field is called as Markov random field in relation to localities system, if for all $z_{i} \in Z$ next condition is realized: $P\left(z_{i} \mid z_{S / i}\right)=P\left(z_{i} \mid z_{N_{i}}\right)$, where $Z=\left\{Z_{1}, \ldots, Z_{m}\right\}-$ random field, $z_{i}-$ values of random variables $Z_{i}$. We use suggestion that OCT-image is Markov Random Field, so pixel intensity depends only from intensities of neighboring pixels. Non-causal locality is used [15]. We estimate autocorrelation function as [31]:

$$
\begin{aligned}
& R(x, y)= \\
& =\sum_{\substack{i=-1 \\
I(x+i, y+j) \in N_{x, y}}}^{\sum^{1} \underset{I(x+i, y+j) \in N_{x, y}}{j=-1}} I(x, y) I(x+i, y+j) .
\end{aligned}
$$

Then we find variation and mean for using them as textural features.

\subsection{Neural networks}

Neural network is a mathematician model that simplifies imitated work of human brain. A standard neural network consists of many simple, connected processors called neurons, each producing a sequence of real-valued activations. Input neurons get activated through sensors perceiving the environment; other neurons get activated through weighted connections from previously active neurons. Some neurons may influence the environment by triggering actions [32]. Neuron is a base element of neural system [33]. It has two types of appendages: with input information 
(dendrites) and with output information (axon, only one for translating impulse to other neurons) [34]. Neural joints between neurons (synapses) play a role of weights to accelerate or slow down impulses. If algebraic sum of impulses exceeds threshold value, then neuron translate impulse to other neurons [35]. Below you can see a mathematician explanation in figure and formulas, where $u_{1}, \ldots, u_{n}$ - input signals; $w_{1}, \ldots, w_{n}-$ synaptic weights; $\mathrm{y}$ - output signal; $\mathrm{v}$ - threshold value.

$$
\begin{aligned}
& y=\left\{\begin{array}{l}
1, \sum_{i=1}^{N} w_{i} u_{i} \geq v \\
0, \sum_{i=1}^{N} w_{i} u_{i}<v
\end{array}\right. \\
& y=f\left(\sum_{i=0}^{N} w_{i} u_{i}\right) ; \\
& f(x)=\left\{\begin{array}{l}
1, x \geq 0 \\
0, x<0
\end{array}\right.
\end{aligned}
$$

where $w_{0}=v ; u_{0}=-1$.

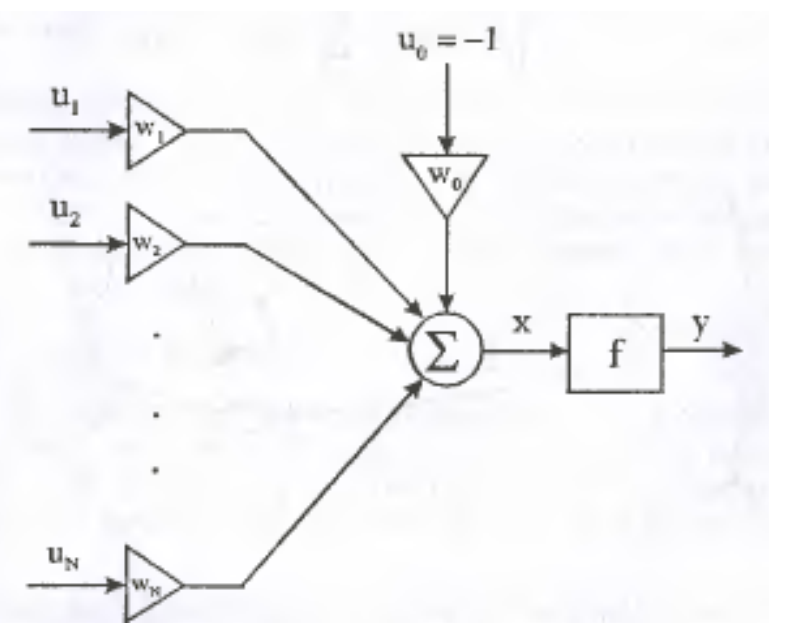

Fig. 4 Model of neuron from artificial neural network [33].

This paper uses scaled conjugate gradient backpropagation [36] based artificial neural network for tissues discrimination (tumor, norm; melanoma, nevus, basalioma or healthy skin) quality assessment. The ANN is trained in MATLAB using 10 corresponding parameters for B-scans as fractal dimensions, counted by $1 \mathrm{D}$-box-counting method (with standard deviation), 2D-differential box counting method and 2D-power spectrum method, Haralick features (contrast, correlation, homogeneity, energy) and Markov random fields features (variance and mean of autocorrelation function estimation). The quantity of hidden layers is 10. For C-scans complex directional features (field variance and weight function variance) were used, the quantity of hidden layers was 20 .

\subsection{Boosting}

Despite the possible good results of classifiers, it is always possible to improve them by linear combination. In our case we use boosting [18]. The task of precedents learning is considered. $\left\langle X, Y, y^{*}, X^{l}\right\rangle$, where $X-$ space of objects; $y^{*}: X \rightarrow Y-$ unknown target dependence; $X^{l}=\left(x_{1}, \ldots, x_{l}\right)-$ is a learning selection; $Y^{l}=$ $\left(y_{1}, \ldots, y_{l}\right)$ is a vector of answers on learning objects $y_{i}=y^{*}\left(x_{i}\right)$. It is needed to build an algorithm: $X \rightarrow Y$, that approximated target dependence $y^{*}$ on all set $X$ [37].

The task of classification on two classes is considered, $Y^{l}=\{-1,+1\}$. We suggest that solution rule is fixed, $C(b)=\operatorname{sign}(b)$.

Base algorithms return answers $-1,0,+1$. Answer $b_{t}(x)=0$ means that base algorithm $b_{t}$ refused from classification object $x$ and answer $b_{t}(x)$ don't use in composition. Finding algorithm composition has look [38]:

$$
\begin{gathered}
a(x)=C\left(F\left(b_{1}(x), \ldots, b_{T}(x)\right)\right)= \\
=\operatorname{sign}\left(\sum_{t=1}^{T} a_{t} b_{t}(x)\right), x \in X
\end{gathered}
$$

This paper uses conjugate AdaBoost [5] algorithm for tissues discrimination (tumor, norma; MM, nevus, $\mathrm{BCC}$ or healthy skin) quality assessment. The boosting for B-scans uses 10 corresponding parameters as fractal dimensions (FD), counted by 1D-box-counting method (with standard deviation (SD)), 2D-differential box counting method and 2D-power spectrum method, Haralick features(contrast, correlation, homogeneity, energy) and Markov random fields features(variance and mean of autocorrelation function estimation). For $\mathrm{C}$-scans complex directional features (field variance and weight function variance) are used, the maximum quantity of trees [39] was 200.

\section{Results and Discussion}

The anisotropic algorithm has been used for denoising. Then images are processed by four methods: Haralick's features, fractal analysis, Markov random fields features (for B-scans) and complex directional field features (for C-scans).

A CCD-sensor in camera is one of noise sources in any acquisition system. The final denoised B-scan and original are shown on Fig. 5. One can see background, edge of skin and inner layers with characterized dark cores ("nests") of BCC. After filtration, the quality of image is visually improved. Moreover, the Signal-toNoise Ratio (SNR) increases 1.4 times in heterogeneous regions to 4 times in background and homogeneous regions.

The Fig. 6 shows a separating healthy skin from MM. The Fisher's linear discriminant analysis has been used in all cases described here [40] and classes are good separated by the linear classificatory. The MMSkin separating achieved $88 \%$ sensitivity and $92.8 \%$ specificity for the contrast-correlation method. In case of correlation-homogeneity and MM-nevus, $88 \%$ sensitivity for linear classificatory, also as $95.2 \%$ specificity have been obtained. 


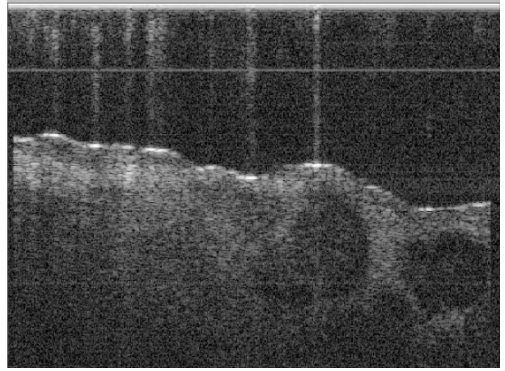

a

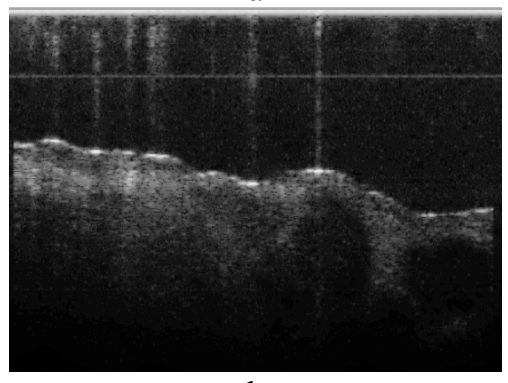

b

Fig. 5 (a) Original B-scan of BCC and (b) B-scan proceeded after filtration.

The Fig. 7(a) shows a case healthy skin versus MM. The sensitivity for variance of AF (MRF) - mean of AF (MRF) is $92.8 \%$, specificity is $95.2 \%$. On the Fig. 7(b) for MM-BCC we have $90.4 \%$ and $83.3 \%$ respectively by using MRF features. One can observe a stronger correlation in this case between features in contrast with Fig. 6. Generally, that means this feature's pair could be reduced to one single feature. But, in the case, an angle between the all data approximation (the "trend") line and Fishers' discriminant one is less than $\pi / 2$, which means both features are particularly valuable.

The Fig. 8 shows us C-scan of skin, the complex directional field of image and weight function. Optical tissue properties changing leads to modification of "sand" texture features can be detected by naked eye also. The Fig. 9 shows dividing MM from BCC and BCC from Nevus. For MM-BCC sensitivity is $91.5 \%$, specificity is $100 \%$. For BCC-Nevus sensitivity is $100 \%$, specificity is $97.5 \%$. This case is also demonstrating a very strong correlation, but, this time, this is an inner class correlation. Both classes are good correlated and, which is much more important, discriminated. This is not correct for small mixed groups on right side of both parts of the Fig. 9. That fact may be explained by a fact MM and BCC tissues could have a very complex topology and a not trivial histology report. In many cases the MM is growing inside a nevus background. Moreover, the MM/BCC could be mixed with other benign tumors. But in our study we choose a binary classes system with no details.

The Fig. 10(a) shows dividing norm from tumor with sensitivity in $86.9 \%$ and specificity in $89.3 \%$ after

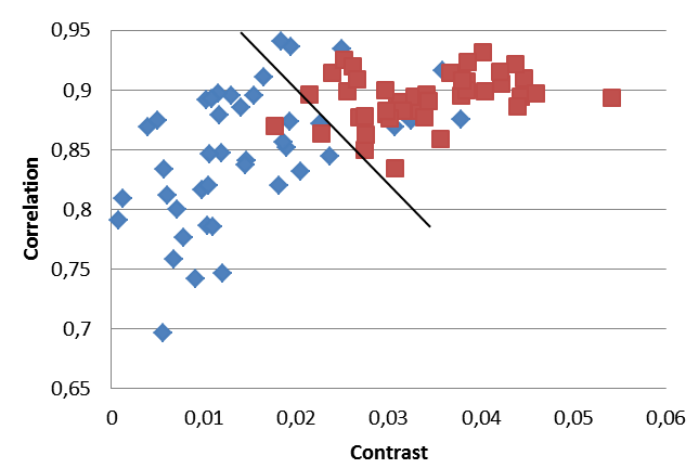

a

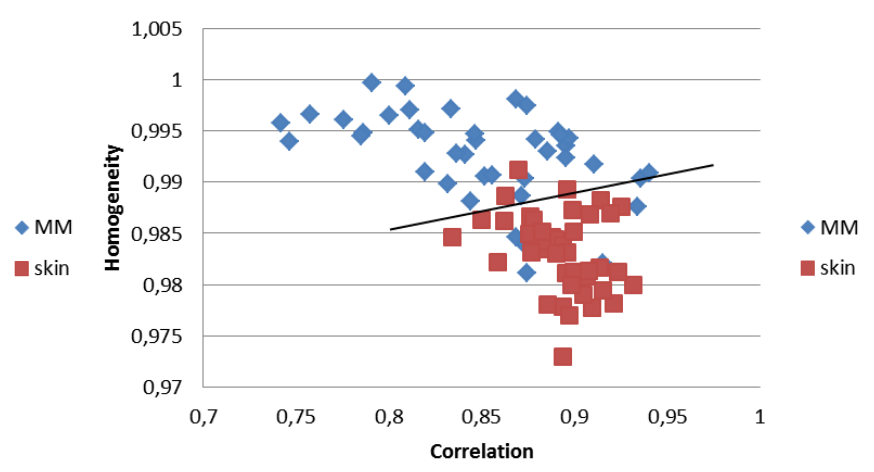

b

Fig. 6 (a) Contrast-Correlation for MM-Healthy Skin and (b) Correlation-Homogeneity for MM-Healthy Skin.

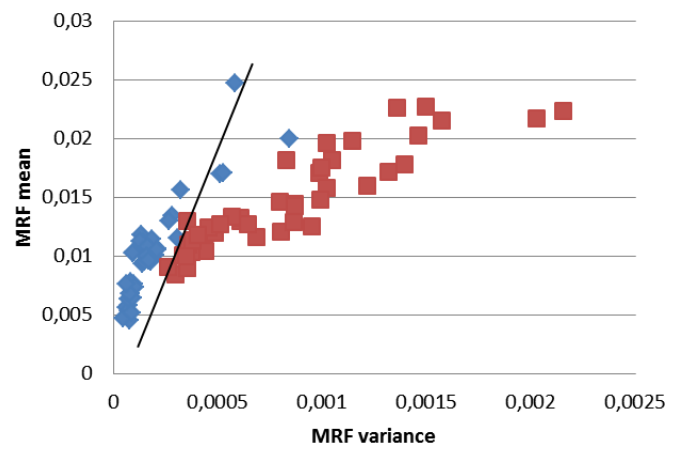

a

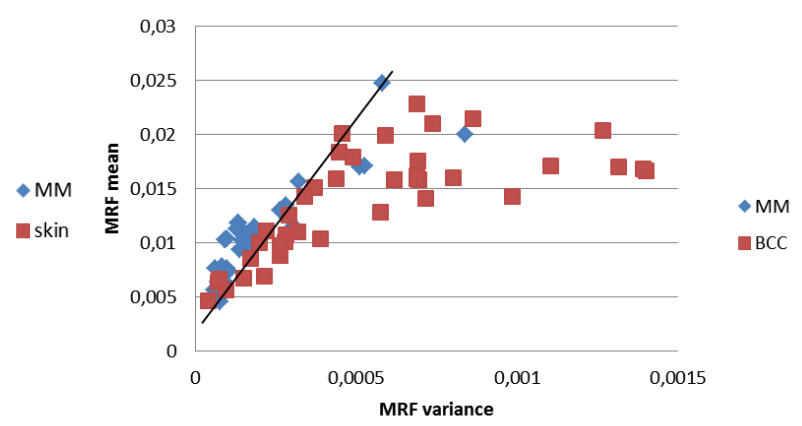

b

Fig. 7 (a) Variance MRF-Mean MRF for MM-Healthy Skin and (b) variance MRF-Mean MRF for MM-BCC. 


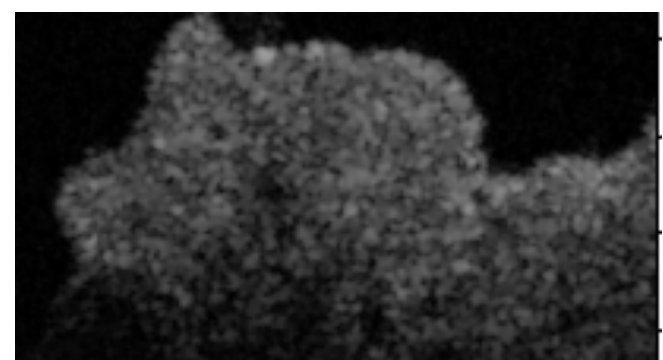

a

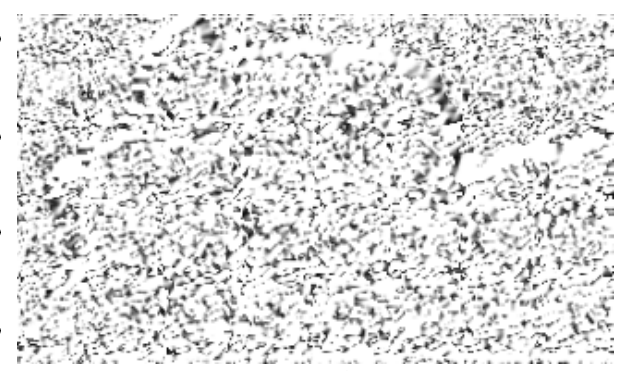

b

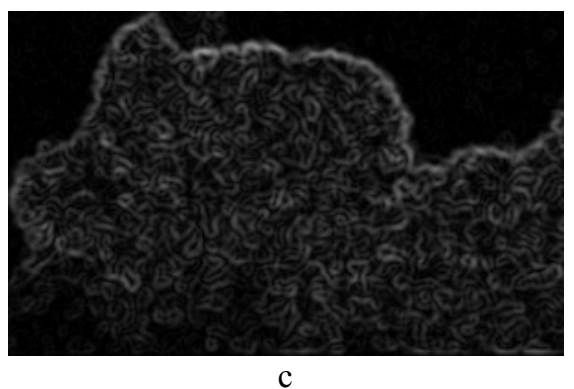

Fig. 8 (a) Original image, (b) complex directional field and (c) weight function [41, 42].

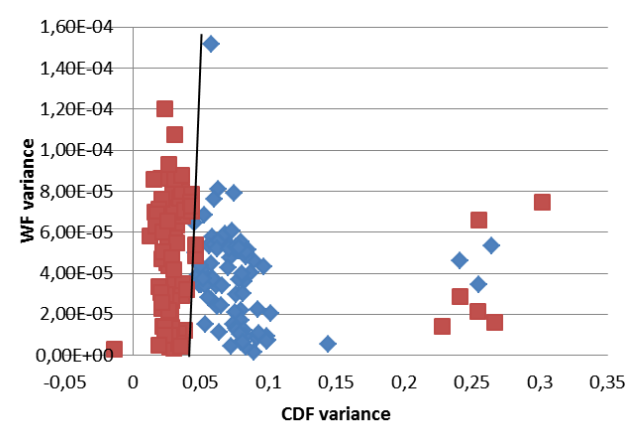

$\mathrm{a}$

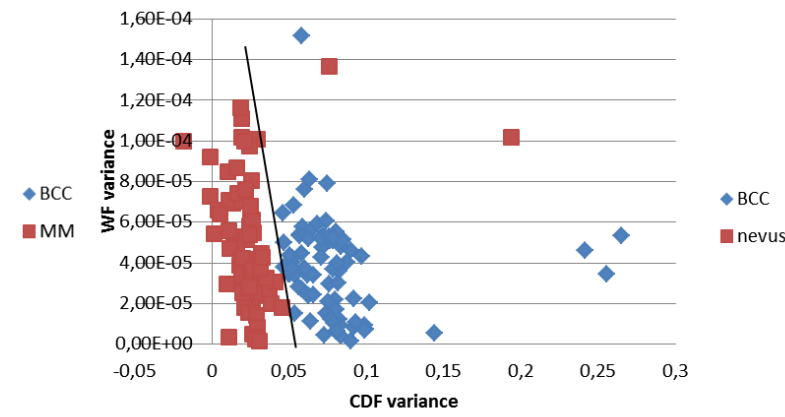

b

Fig. 9 (a) Complex directional field variance - weight function variance for MM-BCC and (b) complex directional field variance weight function variance for $\mathrm{BCC}-$ nevus.
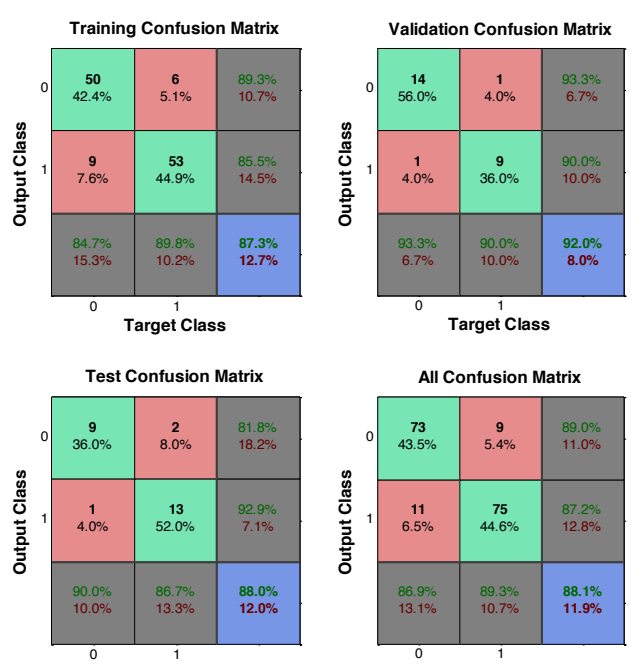

Target Class

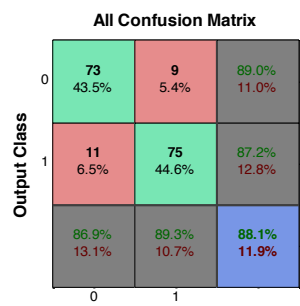

Target Class
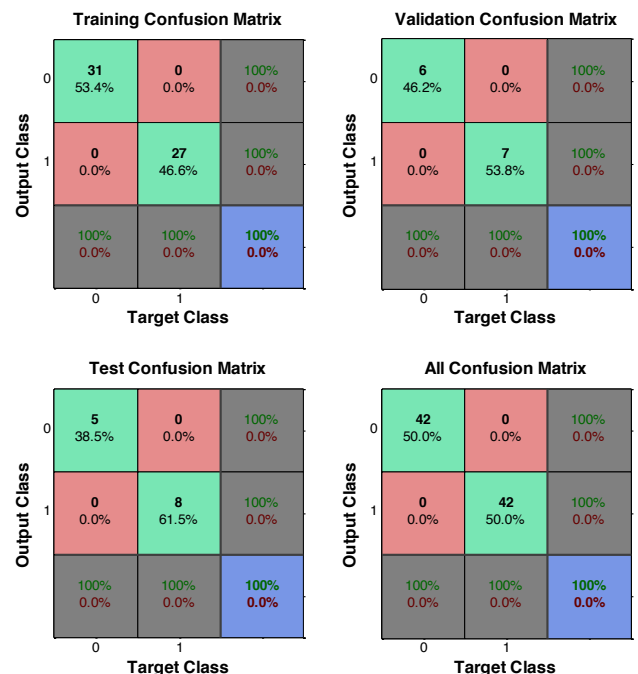

$\mathrm{b}$

Fig. 10 (a) Confusion matrixes for Tumor-Norm and (b) MM-Healthy Skin. 
using neural network with scaled conjugate gradient backpropagation for pattern recognition. The Fig. 10(b) shows dividing MM from healthy skin with sensitivity $100 \%$ and specificity $100 \%$. This incredible result can be explained by a little dataset for ANN in this case, so it should be checked in bigger sets in future investigations

The Fig. 11(a) shows dividing tumor from norm with sensitivity in $73.8 \%$ and specificity in $85 \%$ after
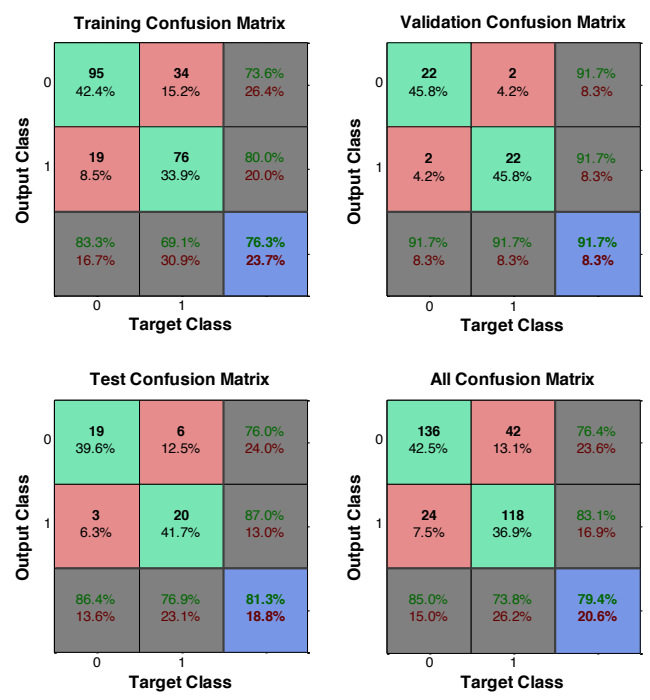

a using neural network with scaled conjugate gradient backpropagation for pattern recognition. The Fig. 11(b) shows classification of species between four classes (MM, BCC, Nevus and Healthy Skin) with precision more than $75 \%$ by using boosting. The summary is presented in Table 1. This amplification is linked with a composition of all features' information, received from tumors and healthy skin on OCT images.

Fig. 11 (a) Confusion Matrix for Norma-Tumor and (b) boosting results for Tumor-Norma case.

Table 1 Statistical characteristics of separation tissues by utilized features.

\begin{tabular}{|c|c|c|c|c|c|c|}
\hline & & \multicolumn{2}{|c|}{ Tissues } & \multirow{2}{*}{$\begin{array}{c}\text { Precision } \\
\text { (sensitivity- } \\
\text { specificity) }\end{array}$} & \multirow{2}{*}{$\begin{array}{l}\text { Quantity } \\
\text { (images) }\end{array}$} & \multirow{2}{*}{$\begin{array}{l}\text { Type } \\
\text { (B/C- } \\
\text { scan) }\end{array}$} \\
\hline $\begin{array}{l}\text { Category of } \\
\text { feature }\end{array}$ & Name of feature & Tissue 1 & Tissue2 & & & \\
\hline Haralick & Contrast-Correlation & MM & Healthy Skin & $88 \%-92,8 \%$ & $42 / 42(84)$ & $\mathrm{B}$ \\
\hline Haralick & Correlation-Homogeneity & MM & Healthy Skin & $88 \%-95,2 \%$ & $42 / 42(84)$ & B \\
\hline MRF & $\mathrm{AF}$ variance- $\mathrm{AF}$ mean & MM & Healthy Skin & $92,8 \%-95,2 \%$ & $42 / 42(84)$ & $\mathrm{B}$ \\
\hline MRF & $\mathrm{AF}$ variance- $\mathrm{AF}$ mean & MM & $\mathrm{BCC}$ & $90,4 \%-83,3 \%$ & $42 / 42(84)$ & B \\
\hline $\mathrm{CDF}$ & $\begin{array}{l}\text { WF variance-CDF } \\
\text { variance }\end{array}$ & Nevus & Healthy Skin & $97,5 \%-83,7 \%$ & $80 / 80(160)$ & $\mathrm{C}$ \\
\hline $\mathrm{CDF}$ & $\begin{array}{l}\text { WF variance-CDF } \\
\text { variance }\end{array}$ & $\mathrm{BCC}$ & Nevus & $100 \%-97,5 \%$ & $80 / 80(160)$ & $\mathrm{C}$ \\
\hline $\mathrm{CDF}$ & $\begin{array}{l}\text { WF variance-CDF } \\
\text { variance }\end{array}$ & MM & $\mathrm{BCC}$ & $91,5 \%-100 \%$ & $80 / 80(160)$ & $\mathrm{C}$ \\
\hline $\begin{array}{c}\text { Neural } \\
\text { networks }\end{array}$ & $\begin{array}{c}\text { Scaled conjugate gradient } \\
\text { backpropogation }\end{array}$ & Tumor & Norma & $86,9 \%-89,3 \%$ & $84 / 84(168)$ & $\mathrm{B}$ \\
\hline $\begin{array}{c}\text { Neural } \\
\text { networks }\end{array}$ & $\begin{array}{c}\text { Scaled conjugate gradient } \\
\text { backpropogation }\end{array}$ & Tumor & Norma & $73,8 \%-85 \%$ & $160 / 160(320)$ & $\mathrm{C}$ \\
\hline Fractal & SD-FD & Tumor & Norma & $70 \%-71 \%$ & $42 / 84(126)$ & $\mathrm{B}$ \\
\hline $\begin{array}{c}\text { Neural } \\
\text { networks }\end{array}$ & $\begin{array}{c}\text { Scaled conjugate gradient } \\
\text { backpropogation }\end{array}$ & MM & $\mathrm{BCC}$ & $100 \%-100 \%$ & $42 / 42(84)$ & $\mathrm{B}$ \\
\hline Boosting & AdaBoost & $\begin{array}{l}\mathrm{MM}, \\
\mathrm{BCC}\end{array}$ & $\begin{array}{l}\text { Nevus, } \\
\text { Healthy Skin }\end{array}$ & $75 \%$ & $42 / 42 / 42 / 42(168)$ & B \\
\hline
\end{tabular}


So after that we can say that all methodologies show good results in classifying melanoma from healthy skin. Boosting and neural networks improve significantly all results for other classifications cases. If we compare boosting and neural networks, then we reveal that ANN shows sensitivity and specificity higher than boosting on same set of B-scans. But boosting let us to divide between four classes (MM, BCC, nevus and healthy skin), but not between 2 classes as other classifiers (for example, Tumor $(\mathrm{BCC}+\mathrm{MM})$ and Norma (Nevus + Healthy Skin). So we receive more universal classifier that can be used with high precision without waiting histological data for diagnostics.

For comparing our results with other works, we should say few words about achievements of other researchers. Gambichler et al. [43] received sensitivity of about $75 \%$ and specificity of about $93 \%$ for MM and nevus in the skin tissue. Multipath OCT system [44] has been successfully used to detect basal cell carcinoma with a sensitivity of $96 \%$ and specificity of $75 \%$. Combined method using OCT and backscatter Raman spectroscopy, characterized in $89-100 \%$ sensitivity and 93-96\% specificity for OCT imaging of skin cancer [45]. Texture analysis and pattern recognition applied to dermoscopy images of malignant melanoma in [46] with a sensitivity of $89 \%$ and specificity of $93 \%$. We also have precision on level more $90 \%$ for some cases, so that is comparable with simplified investigations. Our previous results $[41,42]$ are improved by creation of new more universal classifier, based on boosting and new results for CDF with better precision.

As for diversification of proposed results, utilized features not only give us information about texture of skin and tumor, but indirectly provide geometric characteristics, that can be used by physicians for ABCDE criteria. For example, fractal features help us to evaluate the "B", which means irregularity of lesion's border, complex directional field features in potential could show us the "E", evolving of tumor on series of images with different dates. Markov random fields features and Haralick's features also have probabilistic properties. Implication of different features with different heterogeneous nature let us speak about multimodality of method, that can be amplified by features of statistics analysis technics.

\section{Conclusion}

This research was dedicated to differentiation between tumors and healthy skin on OCT images, using multimodal approach for cancer recognition by evaluation of some textural features. We built universal classifier with $75 \%$ precision for differentiation between four classes (MM, BCC, nevus and healthy skin). The high precision of discrimination between MM and BCC by three methods separately very good corresponds to possibility of visual diagnostics by physicians on OCT images due to a specific form of BCC neoplasm. Also we could note about good results in differentiation between BCC and nevus, MM and healthy skin. The good results by CDF and MRF are very promising to be tested for new cases and new bigger sets of OCT images. The results for MM versus nevus case that were received in our previous work [41] were decreased.

After increasing set of images the best results are on level $93.8 \%$ for sensitivity and $73.8 \%$ for specificity with using MRF. Fractal dimensions, Haralick's and CDF features occurred not effective for this task, neural networks should be used on bigger quantity of images. So we have got to continue our investigations for solving this important challenge. For next investigation new categories of features (morphological, geometrical, statistical, textural etc.) and closer connection with ABCDE criteria (new features for asymmetry, borders irregularity, diameter, evolution) on 2D (C-scans, mostly) and 3D OCT-images should be involved.

\section{Acknowledgements}

This research was supported by the Ministry of Education and Science of the Russian Federation. Authors are thankful to Dr. Wei Gao from Ningbo University of Technology for Matlab code providing for denoising and fractal dimension calculating. 